\title{
Editorials
}

\section{Real world partnership with patients}

In my former role as an NHS Prescribing Adviser, I advised GPs about cost-effective prescribing. Evidence-based considerations went hand in hand with an eye on the drug budget. We routinely performed drug switches with only a passing regard for patients' wishes. Until one day, during a visit to a GP practice, I informed the GP partners that their prescribing for benzodiazepines was considerably higher than the national average. They explained that they had tried to wean their patients off benzodiazepines, in line with local guidelines, but one of their patients reacted badly to the news of his coping strategy being threatened, and committed suicide. Naturally, this had made them somewhat cautious.

Therein lies the problem, our expectations of how things 'should' be, are revealed to us in carefully considered evidence-based guidelines. Meanwhile, our perception of what reality is' confronts us through our lived experience (Box 1). The often opposing demands of following evidence-based guidelines and acting in partnership with patients can leave GPs with the stark choice of either following guidelines or not. ${ }^{1}$ This is likely to result in patients receiving inferior care and GPs feeling legally vulnerable. Is there a way of pragmatically empowering GPs to make the best decisions in complex circumstances in partnership with their patients, and for these decisions to be systematic, transparent, and defendable?

The original intention of evidencebased medicine was to provide a tool for that purpose. ${ }^{2}$ A new way of thinking was proposed in which clinical decisions would be based on scientific evidence. ${ }^{3}$ At first sight this may appear as though evidence dominates other factors in the decision making process, but the original definition of evidence-based medicine focused on evidence as an addition to the existing factors of clinical expertise and the patient's values, needs, and wishes, not a replacement. ${ }^{3}$ Thus, the patient's lived experience was key to the decision making process.

Why then, do we experience a tension between evidence-based guidelines and the patient's circumstances? ${ }^{4-6}$ In the evolution from evidence-based medicine to guidelines there has been a drift away from the original concept. Evidence-based medicine gave clinicians autonomy to make decisions with individual patients, considering their comorbidities and life context, in combination with the best available clinical evidence, without an overt concern about costs. However, guidelines consider single conditions and populations of patients. They reflect a policy perspective with considerations of costs, and reduce clinician autonomy. ${ }^{7.8}$

Thus, in original evidence-based medicine the 'should' of evidence, being consciously applied to individual patients, was closer to the 'is' of the patient's lived experience, and the clinician had autonomy to use judgement to pull these two aspects together in decision making. Whereas, with the development of guidelines and incentivised targets, the population and policy based 'should' is estranged from the patient's lived experience and the clinician lacks the autonomy to bridge the gap.

INTRODUCING THE TRIANGLE OF REALITY

We therefore struggle to reconcile the 'is' and

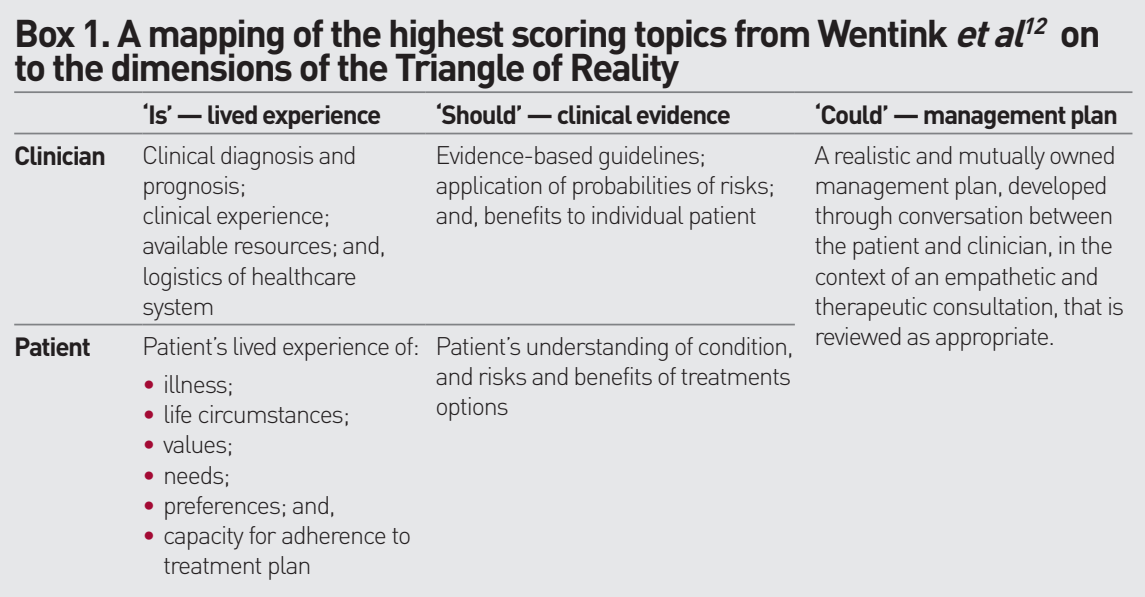

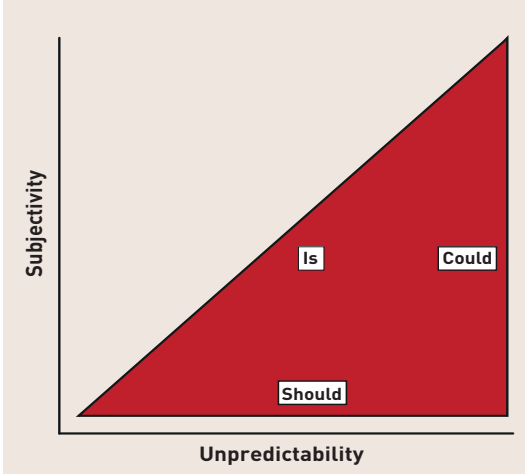

Figure 1. Triangulating reality. Example of application of the Triangle of Reality: A 52 year-old man with pre-diabetes and a BMI of 36 attends his annual health check. He responds defensively to being told to lose weight. The 'is' for the nurse is offering him a 10-minute consultation and a generic healthy eating leaflet. She asks the patient about the 'is' of his living circumstances. He says his over riding concern is debt. He's afraid to open his mail, and eats to alleviate anxiety. He has a good knowledge of diabetes, its complications, and healthy eating (the 'should'). They agree on a 'could' of visiting a debt management service. The nurse appreciates his concern that healthy eating is expensive. They discuss walking to work rather than driving. This helps him to save money, lose weight, improve his mood, and builds trust in the nurse.

the 'should' dimensions of reality. Treatment plans built on the 'is' alone, risk being ineffective or harmful. Treatment plans built on the should'alone, risk being ineffective due to a lack of cooperation with patients in terms of medication adherence or lifestyle changes, or incompatibility with the context. It follows that effective plans should be anchored in both the "is' and the 'should', aiming towards what could be and the collaborative achievement of therapeutic goals. This introduces a third dimension, how things 'could' be, in to our construct of reality. These dimensions can be visualised as a triangle, on axes of subjectivity and unpredictability (Figure 1).

The left-hand corner of the triangle reflects controlled and objective environments, for example, drug development in laboratory conditions. Progressing along the triangle the is' and the 'should' diverge with increasing human involvement, individuality, and therefore subjectivity; accompanied by an increasingly uncontrolled environment and therefore increasing unpredictability. We see this progression moving from the use of drugs in clinical trials, through secondary care, primary care, and finally through to 
public health and screening. Finding a 'could' becomes increasingly problematic as the gap between the is' and the 'should' widens.

By identifying the 'is' and the 'should' it is proposed that GPs and patients can collaboratively work towards a 'could', while recognising the unpredictability of healthcare and life.

Both the clinician and the patient have an 'is' and a 'should' to bring to the consultation. The clinician brings the lived experience of the clinical situation, resources available, and the application of evidence to the individual patient. The patient brings their lived experience and capacity, plus their understanding of the condition and treatment options (see Box 1 and Figure 1).

The integration of clinician and patient dimensions occurs through conversation in the context of an empathetic therapeutic encounter. This process unapologetically requires mastery and time. This may occur as an iterative process of continuing conversation over time in primary care, requiring continuity of care for the best outcomes.? Trust and validation of emotional concerns have been shown to enhance cooperation between clinicians and patients towards achieving targets, but these aspects are not easily measurable and may become overlooked and damaged by over-enforcement of guidelines. ${ }^{10}$

\section{COMPARISON WITH OTHER APPROACHES}

This is a wider approach than shared decision making because it explicitly identifies two dimensions of the patient's agenda (their 'is' and their 'should'), and it actively explores the clinician's lived experience, which includes the logistics of healthcare delivery.

The Triangle of Reality (Figure 1) shows similarities with the patient-centred clinical method and its four interactive components. ${ }^{11}$ The components of 'exploring health, disease, and the illness experience', and 'understanding the whole person' are included in the 'is'. The components of 'finding common ground' and 'enhancing the patient-clinician relationship' are covered by the process of co-creating a 'could'. The Triangle of Reality differs in its explicit discussion of the evidence-base in the should', which includes both the clinician's use of evidence-based guidelines and the patient's understanding of the disease and treatment.

This model offers a tool for patients and clinicians to explicitly explore evidence within the context of their lived experiences, in order to agree on an achievable treatment plan. It aims to restore balance and alignment with the original intention of evidencebased medicine. Establishing effective relationships between clinicians and patients is fundamental for the process of co-creating realistic treatment plans, and primary care should seek to nurture trust and effective communication.

Returning to the example at the beginning, the clinician's should'was to consider the longterm dependency issues of benzodiazepines and the clinician's 'is' was the availability of appointments to call in the patient for a review. The patient's is' was that being on a benzodiazepine provided a crucial coping strategy. It is not known what the patient's understanding of the 'should' was in terms of knowing about the side-effect profile and options for other treatments. Consideration of all of these factors could have led to the provision of an alternative coping strategy of a support group and a slower detox to support the patient through this painful process.

\section{APPLICATION TO DEPRESCRIBING}

The issue of decision making in deprescribing is also explored by Wentink et al in a recent BJGP research paper. They conducted a concept mapping study with 37 patients and 27 professionals to generate factors that were considered to be important for inclusion in decision making about the discontinuation of antidepressant medication. A total of 50 separate topics were generated, which highlights the complexity of these decisions.

As Wentink et al comment: Obviously, reviewing and sharing scientific knowledge is an essential element of clinical decision making that should not be disregarded. However, it may not be the entire story.' That other 'part of the story' is the lived experience, and this is a key consideration in successful decision making in partnership with patients.

The authors call for more concrete tools to aid shared decision making, and their study provides a valuable evidence base for developing a decision aid for the discontinuation of antidepressant medication.

Together these approaches show that supporting holistic consultations, that take into account the best scientific evidence along with the lived reality for both clinicians and patients, in the co-creation of individualised treatment plans offers our best hope of delivering effective medical practice in the face of complexity and uncertainty.

\section{Josie Solomon,}

Deputy Head of School of Pharmacy, University of Lincoln, Joseph Banks Laboratories, Lincoln, UK.

\section{Ruth Shelton,}

Retired Charity Worker and Patient Representative, Nottingham.

\section{Sandy Taylor}

Locum GP, Nottingham.

\section{ADDRESS FOR CORRESPONDENCE}

Josie Solomon

Deputy Head of School of Pharmacy, University of Lincoln, Joseph Banks Laboratories, Lincoln LN6 7DL, UK

\section{Email: JSolomonalincoln.ac.uk}

\section{Provenance}

Freely submitted; externally peer reviewed.

\section{Competing interests}

The authors have declared no competing interests.

DOI: https://doi.org/10.3399/bjgp19X704705

\section{REFERENCES}

1. Solomon J, Raynor DK, Knapp P, Atkin K. The compatibility of prescribing guidelines and the doctor-patient partnership: a primary care mixed-methods study. Br J Gen Pract 2012 DOI: https://doi.org/10.3399/bjgp12X636119.

2. Sackett DL, Rosenberg WM, Gray JA, et al. Evidence based medicine: what it is and what it isn't. BMJ 1996; 312(7023): 71-72

3. Evidence-Based Medicine Working Group. Evidence-based medicine: a new approach to teaching the practice of medicine. JAMA 1992 268(17): 2420-2425.

4. Greenhalgh T, Howick J, Maskrey N, Evidence Based Medicine Renaissance Group. Evidence based medicine: a movement in crisis? BMJ 2014; 348: g3725.

5. Freeman AC, Sweeney K. Why general practitioners do not implement evidence qualitative study. BMJ 2001; 323(7321): 1-5

6. Yudkin JS, Kavanagh J, McCormack JP. Guidelines for treating risk factors should include tools for shared decision making. BMJ 2016; 353: i3147.

7. Chalkidou K, Walley T, Culyer A, et al. Evidenceinformed evidence-making. J Health Serv Res Policy 2008; 13(3): 167-173.

8. Dobrow MJ, Goel V, Upshur RE. Evidence-based health policy: context and utilisation. Soc Sci Med 2004; 58(1): 207-217.

9. Freeman $\mathrm{G}$, Hughes J. Continuity of care and the patient experience. London: The King's Fund, 2010. https://www.kingsfund.org.uk/sites/ default/files/field/field_document/continuitycare-patient-experience-gp-inquiry-researchpaper-mar11.pdf (accessed 18 Jun 2019).

10. Solomon J, Knapp P, Raynor DK, Atkin K. Worlds apart? An exploration of prescribing and medicine-taking decisions by patients, GPs and local policy makers. Health Policy 2013; 112(3): 264-272.

11. Stewart M, Brown JB, Weston W, et al. PatientCentred Medicine: Transforming the Clinical Method. 3rd edn. London: Radcliffe Publishing, 2014.

12. Wentink C, Huijbers, MJ, Lucassen PLBJ, et al. Enhancing shared decision-making about discontinuation of antidepressant medication: a concept mapping study in primary and secondary mental health care. Br J Gen Pract 2019. DOI: https://doi.org/10.3399/ bjgp19X706001. 